\title{
RESISTÊNCIA IN VITRO DE CEPAS DO PLASMODIUM FALCIPARUM ISOLADAS NO SUL DO ESTADO DO PARÁ, EM DIFERENTES PERÍODOS: EMERGÊNCIA DE CASOS DE MULTIRRESISTÊNCIA
}

\author{
A.A. Couto, V.S. Calvosa, M.A. Santos e J.M. Souza
}

\begin{abstract}
O presente estudo avalia a resposta de cepas de Plasmodium falciparum às drogas antimaláricas, através de testes in vitro, isoladas em 7 municípios do sul do Estado do Pará. Foram efetuados 69 testes para cloroquina e mefloquina, 62 para amodiaquina e 61 para quinino. Os resultados mostram elevada resistência para cloroquina (71\%), relativamente baixa resistência para amodiaquina com $(25,8 \%)$ e para o quinino apenas $8,2 \%$. Mefloquina revela ampla sensibilidade (100\%), mas, demonstrando perda da mesma quando comparada em dois periodos distintos. Evidenciou-se também cepas multirresistentes em dois dos municípios estudados.
\end{abstract}

Palavras-chaves: Plasmodium falciparum. Resistência in vitro. Cloroquina. Amodiaquina. Quinino. Mefloquina.

Na Região Amazônica brasileira concentramse mais de $98 \%$ dos casos de malária do país. Segundo Marques ${ }^{6}$, a partir de 1970 , houve uma corrida no sentido da ocupação da Amazônia brasileira, estimulada pela abertura de grandes rodovias, implantação de projetos agropecuários, colonização, construção de hidrelétricas e, em particular, a garimpagem em alta escala, provocando um fluxo migratório, que nos dias atuais ainda é muito intenso, como se verifica no Sul do Estado do Pará.

Conforme os registros oficiais da ex-SUCAMSuperintendência de Campanhas de Saúde Pública, - DECEM - Divisão de Malária, hoje FNSFundação Nacional de Saúde, cerca de $70 \%$ dos casos de malária da Amazônia brasileira ocorrem nos estados de Rondônia e Pará ${ }^{8}$. Neste último, $67,1 \%$ originam-se do sul do estado, onde concentram-se grandes projetos agropecuários, colonização e garimpos ${ }^{13}$.

A população estabelecida em áreas de elevada transmissão de malária no sul do Estado do Pará

\footnotetext{
Programa de Malária, Instituto Evandro Chagas/Fundação Nacional de Saúde (FNS).

Suporte financeiro da FNS, FINEP e UNDP/World Bank/ WHO Special Programme/TRD.

Enderego para correspondência: Dr. Alvaro Couto. Av. Almirante Barroso 492, Marco, 66090-000 Belém, PA. Recebido para publicação em 20/11/92.
}

está constituída por indivíduos procedentes de várias áreas, extrạ e intra Amazônia legal, portanto nãoimunes ou semi-imunes e sem proteção adequada, aliando-se a isso as facilidades no contato vetorhomem na região, levando a ocorrência de malária em elevados níveis e com maior morbidade.

O Plasmodium falciparum é responsável pela maioria dos casos de malária no Estado do Pará $(54,2 \%)$ e também pela mais severa, às vezes fatal, forma da doença. A resistência dessa espécie de parasitoàs drogas antimaláricas convencionais exerce grande ameaça ao efetivo controle da doença. Ela surge, entre outras causas, pelo uso permanente e em grandes quantidades das drogas antimaláricas, utilização de esquemas terapêuticos de forma inadequada, automedicação, possível vantagem biológica dos parasitos resistentes sobre os sensíveis, além de fatores imunológicos ligados ao hospedeiro ${ }^{16}$. Por outro lado, o movimento migratório determina forte influência na emergência e disseminação de cepas resistentes ${ }^{6}$.

A redução da sensibilidade do Plasmodium falciparum aos diversos quimioterápicos tem sido amplamente documentada no Brasil nas últimas duas décadas quer na avaliação in vivo como in vitro 59 1415 . No presente trabalho, procuramos documentar o nível de suscetibilidade de cepas de Plasmodium falciparum para as drogas cloroquina, amodiaquina, quinino e mefloquina em amostras isoladas em sete 
Couto AA, Calvosa VS, Santos MA, Souza JM. Resistência in vitro de cepas do Plasmodium falciparum isoladas no sul do Estado do Pará, em diferentes periodos: emergência de casos de multirresistência. Revista da Sociedade Brasileira de Medicina Tropical 26:5-9, jan-mar, 1993.

municípios do sul do Estado do Pará no período de 1983 a 1986 e incluímos resultados preliminares já divulgados anteriormente 1112 .

\section{MATERIAL E MÉTODOS}

As amostras analisadas no presente estudo foram isoladas de pacientes portadores de malária causada pelo Plasmodium falciparum nos municípios de: Itaituba, Tucuruí, Marabá, Altamira, Redenção do Pará, São Félix do Xingu e na região do alto rio Tapajós, todos com localização no sul do Estado do Pará. Foram tomadas amostras sanguíneas $(2 \mathrm{ml})$ por punção venosa de pacientes com confirmada infecção exclusivamente por Plasmodium falciparum após minuncioso exame hemoscópico bem como presença de parasitemia superior a 500 parasitos por $\mu \mathrm{l}$ de sangue. A amostra sanguínea foi transferida para um tubo de $15 \mathrm{ml}$, contendo meio de cultivo RPMI 1640 (GIBCO) completo e desta forma logo transportado via aérea para nosso laboratório em Belém num prazo nunca superior a 72 horas, conforme metodologia adaptada por Santos e col (dados não publicados).

Para o microteste in vitro, o sangue infectado foi lavado três vezes com meio RPMI 1640 incompleto e submetido a cultivo contínuo, conforme metodologia descrita por Trager e Jansen $^{17}$. Após curto período, até obtenção da parasitemia adequada e com predominância de trofozoítos foram realizados os microtestes segundo método preconizado por Rieckmann e cols ${ }^{10}$.

Para a realização dos testes foram utilizadas microplacas de titulação de polietileno (PETECIL), de fundo chato aplicando-se $0,1 \mathrm{ml}$ de meio completo com concentrações crescentes das drogas nas posições correspondentes. Foi utilizado como controle meio sem droga e todos os testes feitos em duplicatas. Em cada orifício foi então feita a adição de $0,01 \mathrm{ml}$ da suspensão de sangue parasitado oriundo dos cultivos.

As drogas foram diluídas momentos antes da sua utilização nos testes, a partir de uma solução estoque fornecida pela Organização Mundial de Saúde (OMS) e nas concentrações por ela recomendada: difosfato de cloroquina de 1,0 a 32,0 x $10^{-8} \mathrm{M}$; mefloquina de 0,5 a $16,0 \times 10^{-8} \mathrm{M}$; amodiaquina de 0,25 a $16,0 \times 10^{-8} \mathrm{M}$ e quinino de 3,9 a $250,0 \times 10^{-8} \mathrm{M}$.
As placas, contendo parasitos submetidos a diferentes concentrações das drogas, foram incubadas por período de 24 a 48 horas após o que foram confeccionadas gotas espessas para avaliação das parasitemias e interpretação dos resultados, conforme os critérios padronizados pela OMS $^{19}$.

A significância estatística foi avaliada pelo teste do qui-quadrado, tendo como valor crítico $\mathrm{p}=0,05$.

\section{RESULTADOS}

A avaliação da resposta in vitro de amostras do Plasmodium falciparum isoladas nos diferentes municípios do sul do Estado do Pará, para as drogas cloroquina, amodiaquina, quinino e mefloquina mostraram que no período de 1983 a 1986 a resistência à cloroquina já atingia níveis elevados (71\%). No entanto, para a amodiaquina foi evidenciada resistência apenas em $25,8 \%$ das amostras analisadas. Para o quinino, a resistência limitou-se a $8,2 \%$ das amostras, enquanto para a mefloquina observou-se sensibilidade de todas as amostras (Tabela 1).

Tabela 1 - Respostado Plasmodium falciparum in vitro às drogas cloroquina, amodiaquina, quinino e mefloquina em cepas isoladas em diferentes municipios no sul do Estado do Pará, no periodo de 1983 a 1986.

\begin{tabular}{lcrrrr}
\hline Drogas & $N^{0}$ de Testes & $\mathrm{R}$ & \multicolumn{1}{c}{$\%$} & $\mathrm{~S}$ & $\%$ \\
\hline Cloroquina & 69 & 49 & 71,0 & 20 & 29,0 \\
Amodiaquina & 62 & 16 & 25,8 & 46 & 74,2 \\
Quinino & 61 & 5 & 8,2 & 56 & 91,8 \\
Mefloquina & 69 & 0 & 0,0 & 69 & 100,0 \\
\hline
\end{tabular}

A Tabela 2 mostra as repostas do Plasmodium falciparum às drogas estudadas nos municípios do sul do Pará em dois períodos distintos, não se observando diferenças significativas para nenhuma das drogas.

A emergência de cepas multirresistentes foi registrada em Itaituba e Tucuruí para as drogas cloroquina, amodiaquina e quinino. No primeiro município, identificou-se esse fenômeno em uma cepa (IEC. 18/86) e em outras cinco, resistência simultânea à cloroquina e amodiaquina. Para o quinino, somente a concentração considerada limiar da sensibilidade/resistência (125,0pmol/orifício) foi inibitória do crescimento. $\mathrm{O}$ mesmo fenômeno 
Couto AA, Calvosa VS, Santos MA, Souza JM. Resistência in vitro de cepas do Plasmodium falciparum isoladas no sul do Estado do Pará, em diferentes periodos: emergência de casos de multirresistência. Revista da Sociedade Brasileira de Medicina Tropical 26:5-9, jan-mar, 1993.

Tabela 2 - Suscetibilidade do Plasmodium falciparum às drogas cloroquina, amodiaquina, quinino e mefloquina, estratificados em dois periodos: 1983/84 e 1985/86.

\begin{tabular}{lcccc}
\hline & \multicolumn{4}{c}{$N^{\circ}$ de cepas resistentes / total testadas (\%) } \\
\cline { 2 - 5 } Período & cloroquina & amodiaquina & quinino & mefloquina \\
\hline $1883 / 84$ & $36 / 51$ & $13 / 43$ & $02 / 43$ & $00 / 50$ \\
& $(70,6)$ & $(30,2)$ & $(4,7)$ & \\
$1985 / 86$ & $13 / 18$ & $02 / 17$ & $03 / 18$ & $00 / 18$ \\
& $(72,2)$ & $(11,8)$ & $(16,7)$ & \\
\hline
\end{tabular}

ocorreu com a cepa de Tucuruí (IEC. 145/83) (Tabela 3).

Os resultados mostram uma tendência para o aumento da resistência à cloroquina e ao quinino, enquanto que para amodiaquina houve discreta redução desse fenômeno (Tabela 2). Para a mefloquina apesar de não se ter evidenciado resistência em nenhum dos isolados, observou-se uma tendência para menor sensibilidade entre o primeiro e o segundo períodos já que em 1983/84 a sensibilidade, considerando a média das concentrações mínimas inibidoras, era de $\mathrm{CMI}=1,6$ elevando-se para $\mathrm{CMI}=2,3 \mathrm{em} 1985 / 86$ (Figura 1 ).

Tabela 3 - Multirresistência in vitro do Plasmodium falciparum detectada em dois municípios do sul do Estado do Pará, no período de 1983 a 1986.

\begin{tabular}{llccc}
\hline $\begin{array}{l}\text { Locali- } \\
\text { dade }\end{array}$ & Cepa & $\begin{array}{c}\text { R/Clo } \\
\text { pmol }\end{array}$ & $\begin{array}{c}\text { R/Amo } \\
\text { pmol }\end{array}$ & $\begin{array}{c}\text { R/Qui } \\
\text { pmol }\end{array}$ \\
\hline Itaituba & IEC. $128 / 83$ & 32 & 8 & 125 \\
& IEC. $127 / 83$ & 32 & 16 & 125 \\
& IEC. $24 / 85$ & 32 & 8 & 125 \\
& IEC. 8 8/84 & 16 & 8 & 125 \\
& IEC. $20 / 85$ & 32 & 8 & 125 \\
& IEC. $18 / 86$ & 32 & 8 & 250 \\
\hline Tucuruí & IEC. $145 / 83$ & 32 & 8 & 125
\end{tabular}

Limiar da sensibilidade/resistência: cloroquina $8 \mathrm{pmol} /$ orificio; amodiaquina 4 pmol/orificio; quinino $125 \mathrm{pmol} /$ orifício.

\section{DISCUSSÃO}

Este estudo, como parte de um programa amplo de monitoração da resistência do Plasmodium falciparum na Região Amazônica brasileira, através de testes in vitro, permite determinar as tendências

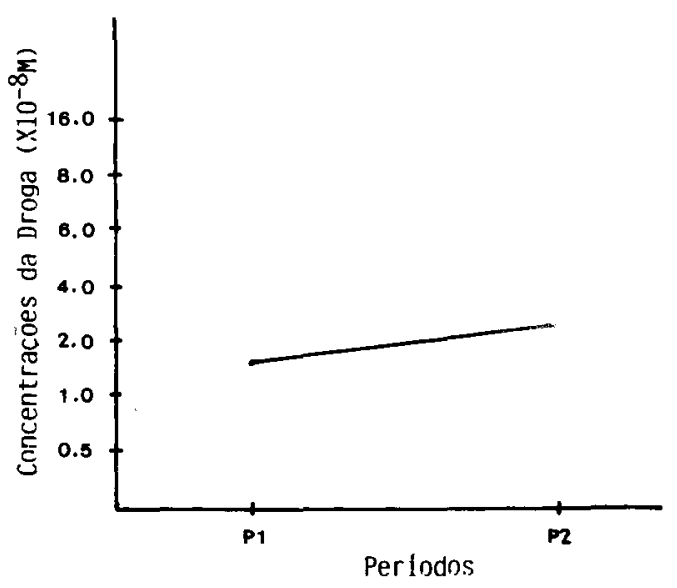

Figura 1 - Redução da sensibilidade à mefloquina entre os periodos 1983/84 (P1) e 1985/86 (P2), avaliada pela média das concentrações mínima inibidora (CMI) entre 68 amostras de Plasmodium falciparum isoladas no sul do Estado do Pará.

temporais e geográficas da resposta desses parasitos às drogas de uso habitual ou em fase experimental. Assim, o nível de resistência do Plasmodium falciparum, determinado pelo microteste in vitro, no presente estudo, revelou $71 \%$ de resistência à cloroquina em concordância com estudos similares desenvolvidos em outras áreas da Amazónia brasileira, que mostram que a resistência à cloroquina está amplamente espalhada na região e em níveis elevados. Vasconcelos e $\mathrm{col}^{18}$, analisando amostras de várias localidades do Estado do Pará registram $67,7 \%$ de resistência; Rosário e col ${ }^{11}$, também no Estado do Pará, 65,3\%; Di Santi e col², em Rondônia, mostram 50\% de cepas cloroquinaresistentes; Santos e $\mathrm{col}^{12}$, analisando amostras dos Estados do Pará, Amapá, Rondônia, Maranhão e Amazonas referem $83 \%$ de cepas resistentes e, mais 
Couto AA, Calvosa VS, Santos MA, Souza JM. Resistência in vitro de cepas do Plasmodium falciparum isoladas no sul do Estado do Pará, em diferentes periodos: emergênicia de casos de multirresistência. Revista da Sociedade Brasileira de Medicina Tropical 26:5-9, jan-mar, 1993.

recentemente, Kremsner ${ }^{4}$ no Acre registra $84 \%$ de resistência a essa droga. Esse fato se agrava, pois, além do elevado nível de resistência observado neste estudo no sul do Estado do Pará e em outras áreas da Região Amazônica, verifica-se uma constante perda de sensibilidade ao longo do tempo para cloroquina. Para amodiaquina observamos, no período estudado, uma ligeira mudança no perfil da resposta para melhor, uma vez que as cepas apresentaram um aumento de sensibilidade. Para o quinino, houve perda de sensibilidade, fato possivelmente associado a maior utilização dessa droga na área.

Mais grave ainda foi a observação de cepas multirresistentes in vitro para as drogas cloroquina, amodiaquina e quinino em dois dos sete municípios avaliados.

A resposta do Plasmodium falciparum para a mefloquina indica alta sensibilidade na área, porém, quando estratificada nos dois períodos, verificou-se uma discreta perda de sensibilidade no segundo período, sugerindo uma tendência à emergência de cepas resistentes a essa droga, provavelmente devido à sua introdução através de vias ilegais e pelo seu uso na Operação Impacto contra a malária a partir de 19867 .

A mobilização da população humana em áreas de alta transmissão da malária traz como conseqüência a facilidade da disseminação de cepas resistentes e esse fato associado a outros fatores, tais como: uso inadequado de medicamentos antimaláricos, automedicação e pressão contínua de drogas, agravam o problema da resistência.

É de fundamental importância, além da determinação quantitativa equalitativa da resistência do Plasmodium falciparum às drogas antimaláricas, elucidar outros fatores epidemiológicos que podem estar, direta ou indiretamente, associados à emergência e disseminação da resistência em uma determinada área ou região como, por exemplo, o regime de uso das drogas antimaláricas.

\section{SUMMARY}

The responses of Plasmodium falciparum to antimalarial drugs were evaluated through the in vitro test using blood sample collected from patients of 7 municipalities of the south of Pará State. Sixty nine microtests for chloroquine and mefloquine, 62 for amodiaquine and 61 for quinine were performed. The results showed a high resistance for chloroquine (71\%), a relatively low resistance level for amodiaquine (25.8\%) and little resistance to quinine (8.2\%). For mefloquine $100 \%$ of sensitivity was found.

Key-words: Plasmodium falciparum. In vitro resistance. Chloroquina. Amodiaquine. Quinine. Mefloquine.

\section{AGRADECIMENTOS}

Os autores agradecem em especial aos integrantes dos Postos de Notificação da extinta SUCAM, nos municípiós em estudo, pela colaboração prestada e a Dra. Antoniana U. Krettli pela análise crítica deste trabalho. Agradecemos também aos auxiliares técnicos de pesquisa do Instituto Evandro Chagas, Srs. Edivaldo Santa Rosa e José Maria Nascimento pela colaboração nas atividades de campo e laboratoriais.

\section{REFERÊNCIAS BIBLIOGRÁFICAS}

1. Couto AA, Santos MM, Calvosa VS. Resistência in vitro do $P$. falciparum em cepas isoladas no Sul do Estado do Pará. In: Resumos do XXV Congresso da Sociedade Brasileira de Medicina Tropical p.270, 1989.

2. Di Santi SM, Boulos M, Vasconcelos MA, Oliveira SG, Couto AA, Rosário VE. Caracterização de cepas de Plasmodium falciparum do Estado de Rondônia, Brasil, utilizando microteste de sensibilidade aos antimaláricos, tipificação enzimática e anticorpos monoclonais. Revista do Instituto de Medicina Tropical de São Paulo 29:142147, 1987

3. Di Santi SM, Camargo Neves VLF, Boulos $\mathbf{M}$, Dutra AP, Ramos AMSV, Santos M, Barata LCB. Avaliação da resposta do Plasmodium falciparum à cloroquina, quinino e mefloquina (1). Revista do Instituto de Medicina Tropical de São Paulo 30:147$152,1988$.

4. Kremsner PG, Zotter GM, Feldmeier H, Bienzle U, Jansen-Rosseck R, Graninger W, Rocha RM, Wernsdorfer WH. Differences in drug response of Plasmodium falciparum within an area of the Amazon region. Transactions of the Royal Society of Tropical Medicine and Hygiene 83:158-161, 1989.

5. Kremsner PG, Zotter GM, Feldmeier H, Graninger W, Kollaritsch M, Wiedermann G, Rocha RM, Wernsdorfer WH. In vitro drug sensitivity of Plasmodium falciparum in Acre, Brazil. Bulletin of the World Health Organization 67:289-293; 1989. 
Couto AA, Calvosa VS, Santos MA, Souza JM. Resistência in vitro de cepas do Plasmodium falciparum isoladas no sul do Estado do Pará, em diferentes periodos: emergência de casos de multirresistência. Revista da Sociedade Brasileira de Medicina Tropical 26:5-9, jan-mar, 1993.

6. Marques AC. Migration and dissemination of malaria in Brazil. Memórias do Instituto Oswaldo Cruz 81(supl II):17-30, 1986.

7. Ministério da Saúde/SUCAM/Diretoria Regional do Pará. Operação Impacto Contra a Malária. Relatório Técnico, 1986.

8. Ministério da Saúde/SUCAM/DECEM/Divisão de Malária. Dados Epidemiológicos da Malária do Brasil, 1970 a 1989.

9. Reyes $\mathrm{S}$, Osanai $\mathrm{CH}$, Passos AD. Resistência in vivo do Plasmodium falciparum as 4-aminoquinoleinas e à associação sulfadoxina + pirimetamina II. Estudo de Manaus, Amazonas 1983-1984. Revista Brasileira de Malariologia e Doenças Tropicais 38:37-44, 1986.

10. Rieckmann KH, Campbell GH, Sax LJ, Mrema JE. Drug sensitivity of Plasmodium falciparum. An in vitro micro technique. Lancet 1:22-23, 1978.

11. Rosário VE, Oliveira SG, Santos MAV, Bellesi N, Couto AA. Caracterização de 15 cepas de Plasmodium falciparum de 8 áreas distintas da região Amazônica brasileira. Revista Brasileira de Malariologia e Doenças Tropicais 38:29-35, 1986.

12. Santos MAV, Couto AA, Oliveira SG, Rosário VE. Estudo in vitro dos níveis de resistência do Plasmodium falciparum a drogas, de 1983 a 1986. Revista do Instituto de Medicina Tropical de São Paulo 29:346-353, 1987.
13. Silva MS, Miléo MFC, Calvosa VSP. Avaliação epidemiológica da malária no Sul do Estado do Pará nos últimos cinco anos. Hiléia Médica 8:83-97, 1987.

14. Souza JM. A phase II clinical trial of mefloquina in brazilian male subjects. Bulletin of the World Health Organization 61:815-820, 1983.

15. Souza JM, Sheth UK, Oliveira RMG, Roulet H, Souza SD. An open randomized phase III clinical trial of mefloquine and quinine plus sulphadoxine + pyrimethamine in the treatment of symptomatic falciparum malaria in Brazil. Bulletin of the World Health Organization 63:603-609, 1985.

16. Target GAT. Chemotherapy and the immune response in parasitic infections. Parasitology 90:661$673,1985$.

17. Trager W, Jansen JB. Human malaria parasites in cultivation continuous. Science 193:674-675, 1976.

18. Vasconcelos MA, Rosário VE. Testes de sensibilidade in vitro de amostras de Plasmodium falciparum da Bacia Amazônica (Brasil). Revista Brasileira de Malariologia e Doenças Tropicais 35:21-28, 1983.

19. World Health Organization. Instructions for the use of the microtest kit for the assessment of the response of Plasmodium falciparum to chloroquine and mefloquine in vitro. Unpublished WHO document MAP/82.1, 1982. 